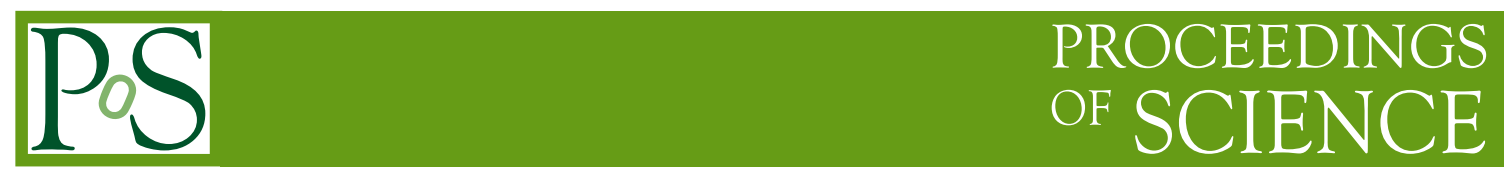

\title{
Spectroscopy of onia and hadrons with open beauty in ATLAS
}

\section{RCW Henderson*}

Physics Department, Lancaster University, Lancaster LA1 4YB, UK

E-mail: $r$.hendersonelancaster.ac.uk

The new observation by ATLAS of an excited open beauty hadronic state, a $B_{c}^{ \pm}$meson with mass $6842 \pm 4$ (stat) \pm 5 (syst) $\mathrm{MeV}$ consistent with expectations for the $B_{c}^{ \pm}(2 S)$ is reported.

The 15th International Conference on B-Physics at Frontier Machines at the University of Edinburgh, 14 -18 July, 2014

University of Edinburgh, UK

\footnotetext{
* Speaker.
} 


\section{Introduction}

The discovery by ATLAS of the $\chi_{b}(3 P)$ onia state [2] is shown and a new observation of an excited $B_{c}^{ \pm}$meson in the second S-wave state $[\bigoplus$ is presented.

\section{The ATLAS detector and spectroscopy}

The general purpose ATLAS detector has contributed significantly to B-physics spectroscopic studies. The Inner Detector with a $2 \mathrm{~T}$ magnetic field has a momentum resolution $\sigma_{p_{T}} / p_{T}=3.8$ $\mathrm{x} 10^{-4} p_{T}(\mathrm{GeV}) \oplus 0.015$ and a pion momentum scale of $\sim 0.1 \%$ at low momentum to $\sim 1 \%$ at $100 \mathrm{GeV}$ [1]. The track parameters are determined by the Inner Detector alone in the analysis here presented, however the ATLAS muon spectrometer is essential for the trigger and provides excellent offline muon identification. It is the success of the B-trigger which means that despite increasing luminosity it is possible to maintain an un-prescaled $J / \psi \rightarrow \mu \mu$ and $\Upsilon \rightarrow \mu \mu$ trigger without any lifetime cuts, even at high luminosity. Also the Inner Detector's excellent vertex resolution ( $30 \mu \mathrm{m}$ transverse and $50 \mu \mathrm{m}$ longitudinally) means that the primary vertex can be accurately reconstructed despite the pileup associated with higher luminosity. Thus ATLAS has high statistics in the di-muon B-physics channels.

\section{Observation of the $\chi_{b}(n P)$ states}

Figures 1 and 2 show the $m\left(\mu^{+} \mu^{-} \gamma\right)-m\left(\mu^{+} \mu^{-}\right)+m(\Upsilon(1 S))$ mass distributions where the $\gamma$ does and does not convert in the Inner Detector respectively. The peaks correspond to the $\chi_{b}(n P) \rightarrow$ $\Upsilon(1 S, 2 S) \rightarrow \mu \mu+\gamma\left(e^{+} e^{-}\right)$decay chain. This was the first observation of the $\chi_{b}(3 P)$ excited onia state centered at a mass of $10.530 \pm 0.005$ (stat) \pm 0.009 (syst) GeV.

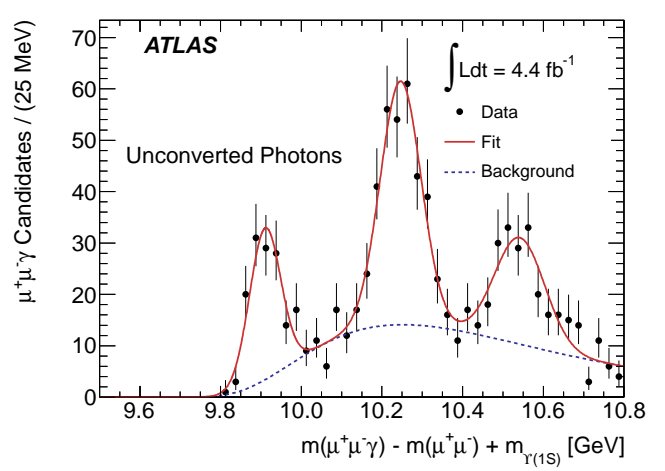

Figure 1: The mass distribution of $\chi_{b} \rightarrow$ $\Upsilon(1 S) \gamma$ candidates for unconverted photons reconstructed from energy deposits in the electromagnetic calorimeter $\left(\chi^{2}\right.$ fit/d.o.f. $\left.=0.85\right)$.

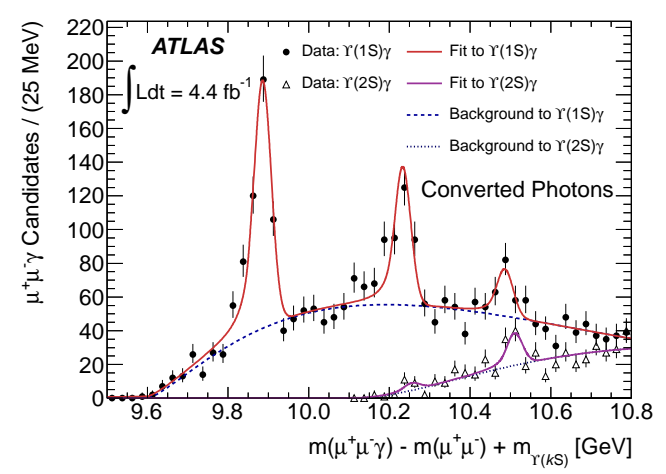

Figure 2: The mass distributions of $\chi_{b} \rightarrow$ $\Upsilon(k S) \gamma(k=1,2)$ candidates formed using photons which have converted and been reconstructed in the ID ( $\chi^{2}$ fit/d.o.f. $\left.=1.3\right)$.

\section{First observation of an excited $B_{c}$ meson}

The ATLAS experiment reports the first observation of an excited $B_{c}^{ \pm}$state [ $\rrbracket$ ], the ground state having been observed in both semi-leptonic and hadronic decay modes. The spectrum and 
properties of $B_{c}^{ \pm}$states are predicted by NRQCD and lattice calculations (see reference 5 in [屯]), so measurements of the ground and excited states will provide tests of the predictions of these models and ultimately provide the opportunity to extract information on the strong interaction potential.

Theory predicts [3] the excited state $B_{c}^{ \pm}(2 S)$ to be in the mass range $6835-6917 \mathrm{MeV}$ and for the pseudo-scalar state to decay via the channel $B_{c}^{ \pm}\left(2 S_{0}\right) \rightarrow B_{c}^{ \pm}\left(1 S_{0}\right)+\pi \pi$. The excited vector state $B_{c}^{ \pm}\left(2 S_{1}\right)$ decays to the excited pseudo-scalar state $B_{c}^{ \pm}\left(2 S_{0}\right)$ via a soft undetectable $\gamma$ so it is included in the same channel.

ATLAS $7 \mathrm{TeV}$ and $8 \mathrm{TeV}$ pp collision data are analysed separately having integrated luminosity of 4.9 and $19.2 \mathrm{fb}^{-1}$ respectively. Monte-Carlo data is treated exactly as collision data; PYTHIA 6 data, tuned for the LHC, are used to generate exclusive $B_{c}^{ \pm}$channels and PYHIA 8 data are used to generate the inclusive $J / \psi X$ channels.

The $B_{c}(2 S)$ candidates are found using kinematic fitting. Firstly a $J / \psi \rightarrow \mu \mu$ candidate is found by fitting two oppositely charged muons to a common vertex. Then a $B_{c} \rightarrow J / \psi+\pi$ candidate is found by fitting the $J / \psi$ and a hadronic track to another secondary vertex. Finally $B_{c}(2 S) \rightarrow B_{c}+\pi \pi$ candidates are formed using two oppositely charged hadronic tracks from the primary vertex, the vertex chosen as the most likely origin of the fitted $B_{c}$ candidate. At each stage all candidate tracks are fully refitted with the muon pair constrained to the $J / \psi$ mass, the previous stages only being used to select candidate tracks. Cuts are made on candidate track $p$ and $p_{T}$ and on the goodness of fit for each vertex. One of the most important cuts requires $\sigma\left(d_{0}\right) / d_{0}$ ( $d_{0}$ being the chosen primary vertex impact parameter) to be high for the hadronic track used to form the $B_{c}(1 S)$ meson candidate, thus removing the bulk of the false candidates. The resulting $B_{c}(1 S)$ meson candidate mass distributions are shown in Figures 3 and 7 for the $7 \mathrm{TeV}$ and $8 \mathrm{TeV}$ data samples respectively. In these figures the solid line shows the projection of the results of the unbinned maximum likelihood fit to all candidates in the mass range $5620-6820 \mathrm{MeV}$. The dashed line is the projection of the background component of the same fit

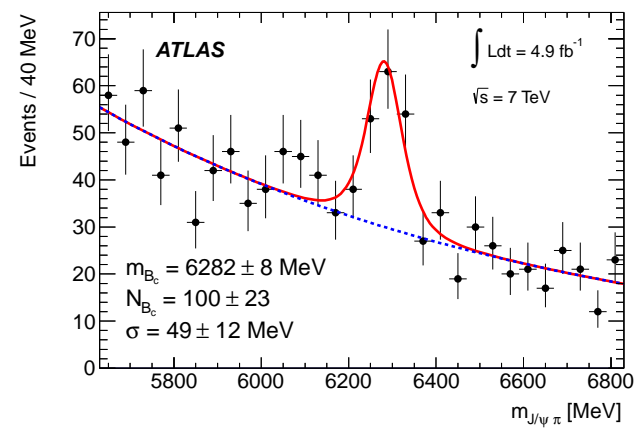

Figure 3: Invariant mass distributions of the reconstructed $B_{c}^{ \pm} \rightarrow J / \psi \pi^{ \pm}$candidates in $7 \mathrm{TeV}$ data.

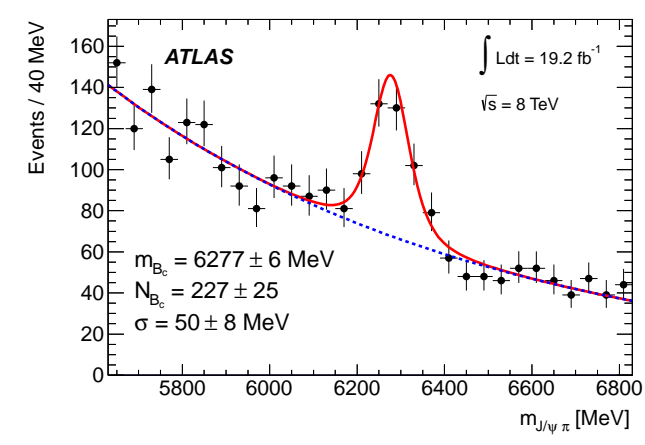

Figure 4: Invariant mass distributions of the reconstructed $B_{c}^{ \pm} \rightarrow J / \psi \pi^{ \pm}$candidates in $8 \mathrm{TeV}$ data.

The quantity $Q=m\left(B_{c}^{ \pm} \pi^{+} \pi^{-}\right)-m\left(B_{c}^{ \pm}\right)-2 m\left(\pi^{ \pm}\right)$is used to find the $B_{c}(2 S)$ candidates. This has the advantage of greatly reducing the effect of resolution of the fitted $B_{c}^{ \pm}$mass. The Figures 5 and 6 below show $Q$ for the $7 \mathrm{TeV}$ and $8 \mathrm{TeV}$ data respectively. In both of these figures the wrong-charge combinations are normalised to the same yield as the right-charge background. The solid line is the projection of the results of the unbinned maximum likelihood fit to all candidates 
in the range $0-700 \mathrm{MeV}$.

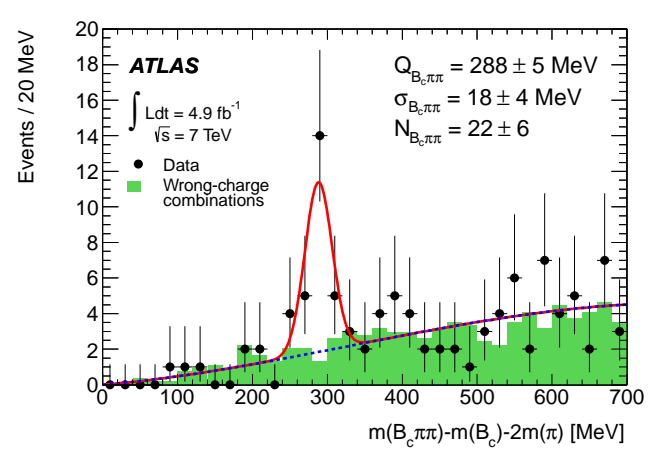

Figure 5: The $Q=m\left(B_{c}^{ \pm} \pi^{+} \pi^{-}\right)-m\left(B_{c}^{ \pm}\right)-$ $2 m\left(\pi^{ \pm}\right)$distribution for the right-charge combinations (points with error bars) and for the same (wrong) pion charge combinations (shaded histogram) in $7 \mathrm{TeV}$ data. The significance of this $B_{c}(2 S)$ signal is $3.7 \sigma$

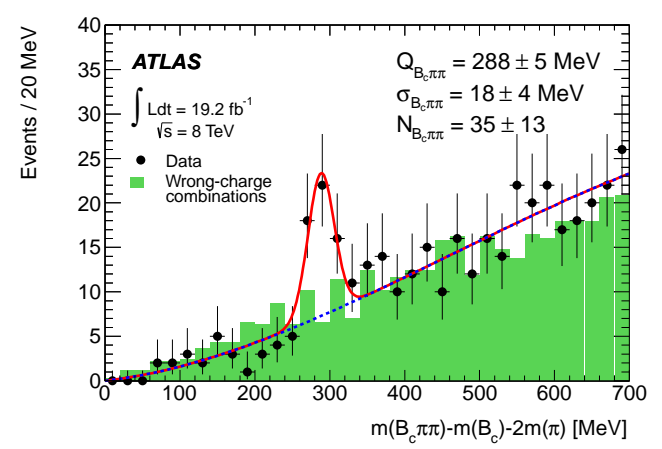

Figure 6: The $Q=m\left(\boldsymbol{B}_{c}^{ \pm} \pi^{+} \pi-\right)-m\left(\boldsymbol{B}_{c}^{ \pm}\right)-$ $2 m\left(\pi^{ \pm}\right)$distribution for the right-charge combinations (points with error bars) and for the same (wrong) pion charge combinations (shaded histogram) in $8 \mathrm{TeV}$ data. The significance of this $B_{c}(2 S)$ this signal is $4.5 \sigma$

The uncertainties shown in these plots are statistical. The systematic uncertainties on the mass of the peak come mainly from two sources. Firstly the mass uncertainty on the $B_{c}$ ground state, which is largely removed by using the $Q$ (mass difference) distribution, and secondly from the fitting of those distributions. The systematics to the $Q$ distribution are investigated by varying the fit background model using either an exponential threshold function, or a higher order polynomial, by varying the fitted mass range from $0-700$ to $0-1500 \mathrm{MeV}$, and by using alternative signal functions such as the Breit-Wigner (BW) function or the BW function convoluted with a Gaussian or double Gaussian. The alternative signal function has negligible effect but the details of the background function and fit range was found to contribute $3.4 \mathrm{MeV}$ and $1.2 \mathrm{MeV}$ respectively to the systematic uncertainty.

The signal significance is determined by generating a large number of toy Monte-Carlo experiments following the background only hypothesis, using the background parameters determined from the fit to the data and scaled to the dataset size. To accommodate the "look elsewhere" effect the fit is not confined to the theoretically expected mass range. The significance is calculated from the fraction of toy experiments for which the difference in the log likelihood ratio with and without a signal component is larger than for collision data. In conclusion a state has been observed with $Q=288.3 \pm 3.5 \pm 4.1 \mathrm{MeV}$ corresponding to a mass of $6842 \pm 4 \pm 5 \mathrm{MeV}$. Its significance for the combined 7 and $8 \mathrm{TeV}$ data is $5.2 \sigma$. This state is consistent with the NRQCD and lattice QCD predictions for the $B_{c}^{ \pm}(2 S)$ excited open beauty meson and so provides a test of those models.

\section{References}

[1] ATLAS Collaboration (2008) JINST 3 S08003

[2] ATLAS Collaboration (2012) Phys. Rev. Lett. 108152001

[3] A.V.Berezhnoy et al (1997) Phys.Atom.Nucl. 60 1729-1740

[4] ATLAS Collaboration (2014) Phys. Rev. Lett. 113212004 